\title{
GALACTIC STRUCTURE WITH THE APS CATALOG OF THE POSS I
}

\author{
J.A. LARSEN \\ University of Minnesota
}

\section{Introduction}

The maturity of all-sky surveys hold great promise for galactic structure studies. Surveys such as the APS Catalog of POSS I allow large scale studies of stellar distributions where the data is very complete to a reasonably faint magnitude, uniformly reduced, and independently calibrated. The APS data covers a large portion of the sky, allowing studies where the primary source of uncertainty is no longer small-number statistics.

Figure 1 shows the locations and coverage of the APS Galactic Structure Fields. These fields are 16 square degree patches of the sky placed every 10 degrees in galactic latitude and 45 degrees in galactic longitude. The survey is designed to probe the Galaxy as Kapteyn suggested, but in a more statistically significant manner. This article presents APS results to date and discusses future work in which all-sky surveys will be valuable.

\section{Galactic Structure from the APS Catalog}

The distribution of stars outside of the Galaxy's disk has been studied. Larsen and Humphreys (1994) have used counts from seven POSS I fields, 16 square degrees each, including the NGP plus six in the $l=90^{\circ} / 270^{\circ}$ plane with $|b|=40^{\circ}$ and $50^{\circ}$. The the ratio of blue stars $(O-E<1.2)$ between these fields has a mean value of 0.5 . If one assumed only halo stars were present a value $c / a \leq 0.5$ for a deprojected $r^{1 / 4}$ law would be derived. When corrected for possible contamination by quasars and a standard model of a thick disk, the ratio increases to only 0.6 . We concluded from this that there is a significant population of stars above the galactic plane in a flattened distribution in addition to those already ascribed to an 


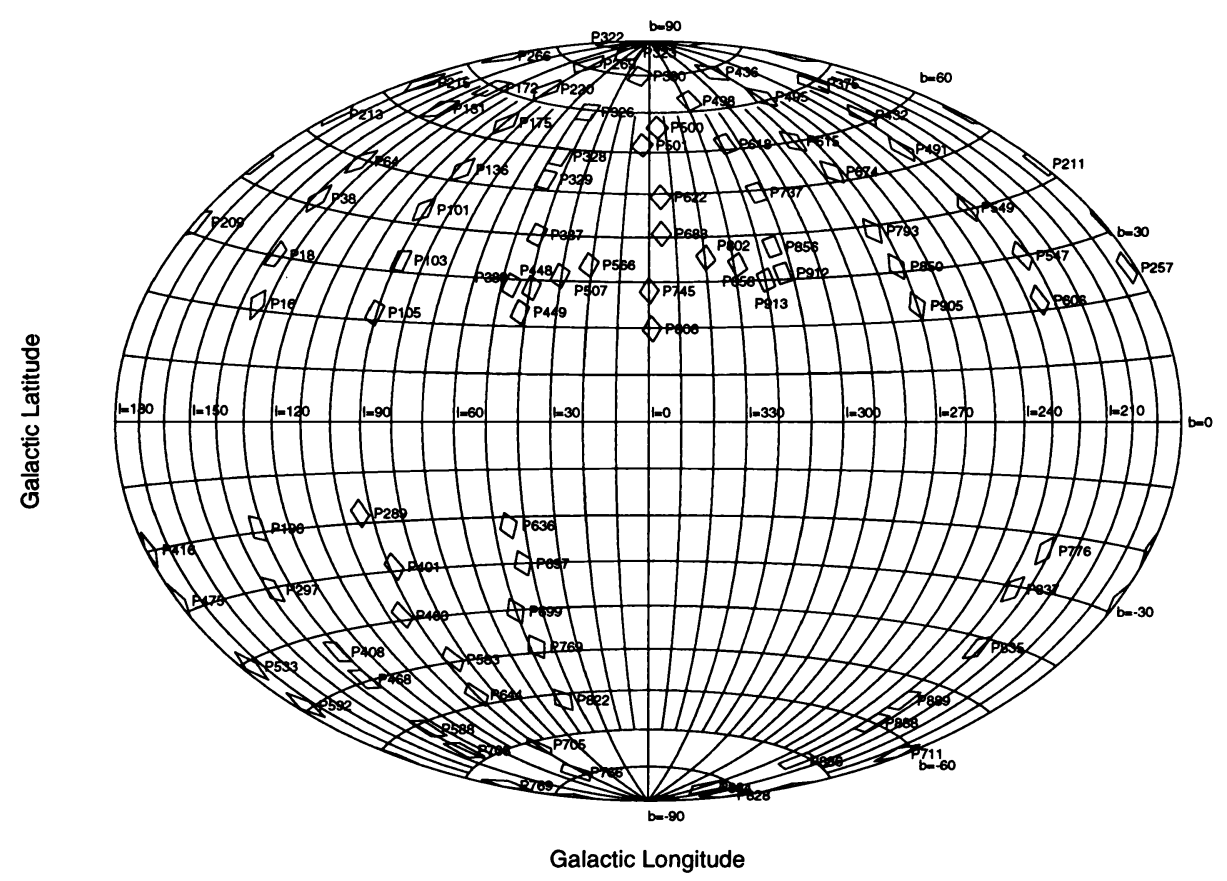

Figure 1. The APS Galactic Structure Fields.

extended or thick disk. Whether this excess represents halo or additional thick disk stars has yet to be determined.

APS counts have been used to determine the Sun's distance $\left(Z_{\odot}\right)$ above the galactic plane (Humphreys and Larsen 1995). Star counts were taken in 12 Palomar Sky Survey fields - six each at the North and South galactic poles-192 square degrees. All stars with $15<O<18$ and $O-E>1.8$ were selected to isolate a disk population. The total counts show significantly more stars in the six fields at the SGP indicating that the Sun is above the galactic plane as defined by neutral hydrogen. The observed ratio of $\mathrm{N}(\mathrm{SGP}) / \mathrm{N}(\mathrm{NGP})$ is $1.11 \pm 0.02$ implying $Z_{\odot}=20.5 \pm 3.5 \mathrm{pc}$.

A large and significant asymmetry has been found in the catalog (Larsen and Humphreys 1996). There are more faint stars in the first quadrant $\left(l=20^{\circ}-45^{\circ}\right)$ of the Galaxy compared to complementary longitudes on the other side (fourth quadrant) of the center-anticenter line. The stars were chosen to be bluer than $B-V \approx 0.6 \mathrm{mag}$ and are most likely subdwarfs. Under this assumption, they are on average approximately $3 \mathrm{kpc}$ away in the direction and at the same distance as the stellar bar proposed by Weinberg (1992) but 1.7 kpc above it. Possible explanations for this excess include an elliptical thick disk, disk heating from an interaction with 
the Sagittarius dwarf, or heating of the disk by the bar. Explaining this asymmetry will give important insights into the early Galaxy.

\section{Future Work}

The APS data has been useful as an exploratory data set. The asymmetry in counts appeared only on the largest spatial scales (across several plates). It was also detected in a region of the sky where our current understanding of galactic structure told us we should not find an asymmetric distribution of stars. The presence of such an asymmetry should serve as ample warning that fundamental galactic structure parameters cannot be reliably determined from only a handful of directions.

In analyzing the full set of APS Galactic Structure Fields, a new methodology is being applied which can be applied to an arbitrary number of directions. A fast, optimized galaxy model suited for multi-field work was created and a robust global optimization routine (the genetic algorithm) was applied for the first time to modeling the Galaxy. From these tools, a set of parameters matching the APS star counts better than extant models was self-consistently derived from the data. These initial studies not only recover the asymmetry, but also several other interesting features.

At the Formation of the Galactic Halo.... Inside and Out conference it became quite obvious that all-sky surveys are necessary for an understanding of the structure of our Galaxy. The current consensus seems to be that both the Eggen, Lynden-Bell, and Sandage (1962) rapid collapse and the Searle and Zinn (1978) merger processes probably played a role in forming the galactic halo. If true, "pencil-beam" surveys can no longer be relied on to provide an understanding of any more than the local environment of the halo. All-sky surveys are essential to providing the context in which the more detailed small surveys can be interpreted.

\section{References}

Eggen, O.J., Lynden-Bell, D., and Sandage, A.R. 1962 Astrophys.J., 136, 748

Humphreys, R.M. and Larsen, J.A. 1995 Astron.J. 110, 2183

Larsen, J.A. and Humphreys, R.M. 1994 Astrophys.J.Lett., 436, L149

Larsen, J.A. and Humphreys, R.M. 1996 Astrophys.J.Lett., in press

Searle, L. and Zinn, R. 1978 Astrophys.J. 225, 357 